\title{
Imatinib mesylate and nilotinib decrease synthesis of bone matrix in vitro
}

\author{
LYSANN MICHAELA KROSCHWALD ${ }^{1,2}$, JOSEPHINE TABEA TAUER ${ }^{3}$, SONJA INGRID KROSCHWALD ${ }^{4,5}$, \\ MEINOLF SUTTORP $^{6}$, ANNE WIEDENFELD ${ }^{1}$, STEFAN BEISSERT $^{1}$, ANDREA BAUER $^{1}$ and MARTINA RAUNER ${ }^{7}$ \\ ${ }^{1}$ Department of Dermatology, Medical Faculty; ${ }^{2}$ Centre for Translational Bone, Joint and Soft Tissue Research, Medical Faculty, \\ Technical University Dresden, D-01307 Dresden, Germany; ${ }^{3}$ Department of Pediatrics, Shriners Hospital for Children, \\ McGill University, Montreal QC H4A 0A9, Canada; ${ }^{4}$ Simon Alberti Group, Max Planck Institute of Molecular Cell Biology \\ and Genetics, D-01307 Dresden, Germany; ${ }^{5}$ Institute for Biochemistry, ETH Zurich, CH-8093 Zurich, Switzerland; \\ ${ }^{6}$ Department of Pediatrics, Division of Pediatric Hematology and Oncology, Medical Faculty; ${ }^{7}$ Department of \\ Medicine III and Center for Healthy Aging, Medical Faculty, Technical University Dresden, D-01307 Dresden, Germany
}

Received August 23, 2018; Accepted May 13, 2019

DOI: $10.3892 / \mathrm{ol} .2019 .10518$

\begin{abstract}
Tyrosine kinase inhibitors (TKIs), such as imatinib (IMA) and nilotinib (NIL), are the cornerstone of chronic myeloid leukemia (CML) treatment via the blockade of the oncogenic $B C R-A B L 1$ fusion protein. However, skeletal side effects are commonly observed in pediatric patients receiving long-term treatment with IMA. Additionally, in vitro studies have shown that IMA and NIL alter vitamin D metabolism, which may further impair bone metabolism. To determine whether TKIs directly affect bone cell function, the present study treated the human osteoblastic cell line SaOS-2 with IMA or NIL and assessed effects on their mineralization capacity as well as mRNA expression of receptor activator of nuclear factor $\kappa \mathrm{B}$ ligand (RANKL) and osteoprotegerin (OPG), two cytokines that regulate osteoclastogenesis. Both TKIs significantly inhibited mineralization and downregulated osteoblast marker genes, including alkaline phosphatase, osteocalcin, osterix, as well as genes associated with the pro-osteogenic Wnt signaling pathway; NIL was more potent than IMA. In addition, both TKIs increased the RANKL/OPG ratio, which is known to stimulate osteoclastogenesis. The present results suggested that the TKIs IMA and NIL directly inhibited osteoblast differentiation and directly promoted a pro-osteoclastogenic environment through the RANKL-OPG signaling axis. Thus, we propose
\end{abstract}

Correspondence to: Professor Martina Rauner, Department of Medicine III and Center for Healthy Aging, Medical Faculty, Technical University Dresden, Fetscherstr. 74, D-01307 Dresden, Germany

E-mail: martina.rauner@ukdd.de

Key words: imatinib, nilotinib, bone, receptor activator of nuclear factor $\kappa \mathrm{B}$ ligand, osteoblastogenesis, chronic myeloid leukemia that future work is required to determine whether the bone health of CML patients undergoing TKI-treatment should be routinely monitored.

\section{Introduction}

The tyrosine kinase inhibitors (TKI) imatinib (IMA) and nilotinib (NIL) have significantly improved the treatment of chronic myeloid leukemia (CML), Philadelphia-chromosome positive acute lymphoblastic leukemia ( $\mathrm{Ph}+\mathrm{ALL})$, gastrointestinal stromal tumors (GIST), and other malignant diseases. The molecular target of IMA is the oncogenic tyrosine kinase (TK) BCR-ABL1, which is necessary and sufficient to develop CML. IMA was licensed for the treatment of adults in the year 2001 (1-7) and without age restriction in 2003 (8). However, leukemic cells were quickly found to develop resistance or intolerance to IMA therapy (9), which led to the development of NIL (Tasigna ${ }^{\circledR}$, Novartis, Basel, Switzerland), a second generation TKI. NIL is an aminopyrimidine-derivative based on IMA, and has an increased potency and selectivity for the oncogenic BCR-ABL1 kinase, leading to a 20- to 50-fold higher inhibitory activity in IMA-sensitive cells and 3 to 7 times higher inhibitory activity in IMA-resistant cells (10). NIL was approved for the treatment of adult patients insensitive to IMA in 2008 (11).

IMA and NIL exhibit several off-target effects on non-oncogenic TKs, such as platelet derived growth factor receptor (PDGF-R) and colony stimulating factor 1 receptor (CSF1R, c-FMS), which are involved in the bone remodeling cycle (12). IMA impairs bone metabolism in CML patients under prolonged treatment (13-15) as demonstrated by growth retardation in up to $73 \%$ of pediatric $\mathrm{CML}$ patients (12). Furthermore, CML patients undergoing IMA treatment, frequently experience hypophosphatemia, which is associated with reduction in serum 25-hydroxyvitamin $\mathrm{D}_{3}$ (calcidiol) and 1.25-dihydroxyvitamin $\mathrm{D}_{3}$ (calcitriol) (16). Calcitriol regulates a wide variety of genes associated with bone formation, as demonstrated by the role of calcidiol in 
promoting mineralization and reducing osteoblast proliferation and osteoclast differentiation. In vitro studies have demonstrated that IMA and NIL interact with the vitamin D metabolism pathway by competitively inhibiting CYP27B1, the enzyme involved in hydroxylating calcidiol to its active form calcitriol $(17,18)$. However, the underlying pathophysiological mechanisms remain poorly defined.

Bone formation is carried out by osteoblasts producing bone matrix and mineral crystals whereas bone resorption is carried out by osteoclasts resorbing bone matrix through proteolytic enzymes and acidic dissolution of the minerals. One of the key pathways regulating osteoclastogenesis is the receptor activator of nuclear factor $\kappa \mathrm{B}$ ligand (RANKL) pathway. Its receptor RANK is expressed on osteoclast precursors. Upon binding of RANKL, osteoclast differentiation is initiated through the activation of specific downstream signaling pathways.

Osteoprotegerin (OPG) functions as a decoy receptor for RANKL and prevents binding of RANKL to its receptor RANK, consequently serving as a negative regulator of osteoclastogenesis. Thus, the RANKL/OPG ratio is an essential determinant of bone mass and skeletal integrity (19). Calcitriol and other hormones such as parathyroid hormone (PTH) control the expression of RANKL. As TKIs are known to interfere with vitamin D metabolism and suppress longitudinal growth in children, we investigated whether TKIs exert direct effects on osteoblasts and the RANKL cascade in vitro.

\section{Materials and methods}

Cell culture. Human osteosarcoma cells (SaOS-2; Leibniz Institute DSMZ-German Collection of Microorganisms and Cell Cultures, Braunschweig, Germany) were seeded at a density of $1 \times 10^{5}$ cells $/ \mathrm{cm}^{2}$ and grown in a humidified $5 \% \mathrm{CO}_{2}$ atmosphere at $37^{\circ} \mathrm{C}$. For proliferation McCoy's 5A medium without phenol red (BioConcept, Allschwil, Switzerland) supplemented with $15 \%$ fetal bovine calf serum and $1 \%$ Penicillin and Streptomycin was used. Ten days after seeding, osteogenic differentiation was induced by growing the cells in Alpha Minimal Essential Medium supplemented with $15 \%$ fetal bovine calf serum, $10 \mathrm{mM} \beta$-glycerophosphate, $2 \mathrm{mM}$ L-glutamine, $300 \mu \mathrm{M}$ L-ascorbic acid 2-phospate (all from Sigma Aldrich, Germany) and 1\% Penicillin and Streptomycin.

For hemacolor and immunofluorescence staining cells were grown in 12-well-plates on cover slips ( $\varnothing 13 \mathrm{~mm}$ ), while for RNA analyses, cells were grown in culture flasks for up to 25 days. Medium was changed 3 times a week. IMA or NIL (Novartis, Basel, Switzerland) was added to the cell culture medium at a concentration of $1 \mu \mathrm{M}$, respectively. After 5,10 , 15 or 25 days of incubation, mineralization capacity and the RANKL cascade were assessed.

Cell staining and microscopy. Hemacolor staining (Biomed, Oberschleißheim, Germany) was performed according to the manufacturer's instructions. After staining, samples were mounted with Celltexx (Engelbrecht Medizin und Labortechnik $\mathrm{GmbH}$, Edermünde, Germany). Digital images were obtained using an Axio Imager A1 (Zeiss, Jena, Germany).
Immunofluorescence staining and confocal microscopy. Cells were washed with PBS, fixed with $4 \%$ paraformaldehyde (Roth, Karlsruhe, Germany), and stored in PBS at $4^{\circ} \mathrm{C}$. All washing steps were performed twice for $10 \mathrm{~min}$ in PBS-T [PBS supplemented with $0.005 \%$ Tween (Serva, Heidelberg, Germany)]. Cells were stained with the following primary antibodies: RANKL mouse IgG2B, RANK mouse IgG2A and OPG mouse IgG1 (R\&D, Minneapolis, USA). The respective secondary antibodies Alexa Flour ${ }^{\circledR} 546$ Goat anti-mouse IgG2b, Alexa Flour ${ }^{\circledR} 488$ Goat anti-mouse IgG2A and Alexa Fluor ${ }^{\circledR} 647$ Goat ant-mouse IgG1 (Thermo Fisher Scientific, Waltham, USA) were applied for detection. The primary antibodies [dissolved in PBS supplemented with $0.001 \%$ Tween and $1 \%$ BSA (Sigma-Aldrich, Steinheim, Germany)] were applied at $8 \mu \mathrm{g} / \mathrm{ml}$ over night at $4^{\circ} \mathrm{C}$. After washing the secondary antibody (diluted 1:200) was applied for $1 \mathrm{~h}$. After another washing step, DAPI (Sigma-Aldrich, Steinheim, Germany) staining was prepared at $1 \mu \mathrm{g} / \mathrm{ml}$ in PBS and applied for $5 \mathrm{~min}$ in the dark at room temperature followed by a 1 min PBS wash. Finally, the samples were mounted with Fluorescence Mounting Medium (DAKO, Jena, Germany).

Fluorescence microscopy was performed using a DeltaVision microscope system composed of softWoRx 5.5.1 software (Applied Precision, United Kingdom), Olympus IX71 microscope (Olympus, Tokyo, Japan), Cool SnapHQ camera (Photometrics, Tucson, Arizona) and Plan Apo N 60x1.4 numerical oil objective (Nikon, Tokyo, Japan). Cell boundaries were observed by thresholding the bright-field image and overlaying it with the fluorescence image. $Z$ stacks with 10 focal planes were collected at 7 regions of interest for each sample and each channel (DAPI, FITC, TRITC, CY5). Analysis of images and measuring of fluorescence intensity were performed manually using Fuji software (FujiFilm, Tokyo, Japan) (20). Z projection was done using average intensity projection.

RNA isolation and quantification. RNA was extracted using the RNeasy ${ }^{\circledR}$ Plus Mini Kit (Qiagen, Hilden, Germany) according to the manufacturer's instructions. RNA was dissolved in $20 \mu \mathrm{l}$ RNase-free water (Invitrogen, Carlsbad, USA) and concentrations were measured by an Infinite ${ }^{\circledR} 200$ Pro plate reader (Tecan, Männedorf, Switzerland). Samples with a 260/280 ratio of $\geq 2$ were classified as pure RNA and used for further investigations.

Reverse transcription of $0.5 \mu \mathrm{g}$ template RNA with random hexamer primers was carried out using iScript cDNA Synthesis kit (Bio-Rad Lab, Munich, Germany) according to the manufacturer's instructions. Reverse transcription-quantitative PCR was performed in $20 \mu 1$ reaction volumes containing $0.5 \mathrm{ng} / \mu \mathrm{l}$ of diluted cDNA, $10 \mu \mathrm{l}$ of iQ SYBR Green Supermix (Bio-Rad Lab, Munic, Germany), $0.6 \mu 1$ of $10 \mathrm{pmol} / \mu 1$ forward primer, and $0.6 \mu \mathrm{l}$ of $10 \mathrm{pmol} / \mu 1$ reverse primer. A Stratagene MX300P cycler (SABiosciences, Qiagen, Hilden, Germany) was used with the following profile: $95^{\circ} \mathrm{C}$ for $3 \mathrm{~min}$ followed by 40 cycles of $95^{\circ} \mathrm{C}$ for $15 \mathrm{sec}, 60^{\circ} \mathrm{C}$ for $30 \mathrm{sec}$, and $30 \mathrm{sec}$ at $72^{\circ} \mathrm{C}$ with one final cycle of $55^{\circ} \mathrm{C}$ for $1 \mathrm{~min}$ and $30 \mathrm{sec}$ at $95^{\circ} \mathrm{C}$. The results were calculated using the $\Delta \Delta \mathrm{Cq}$ method and presented as fold increase relative to GAPDH expression.

Primers were purchased from MWG Biotech AG (Ebersberg, Germany). The sequences were: RANKL, forward: 


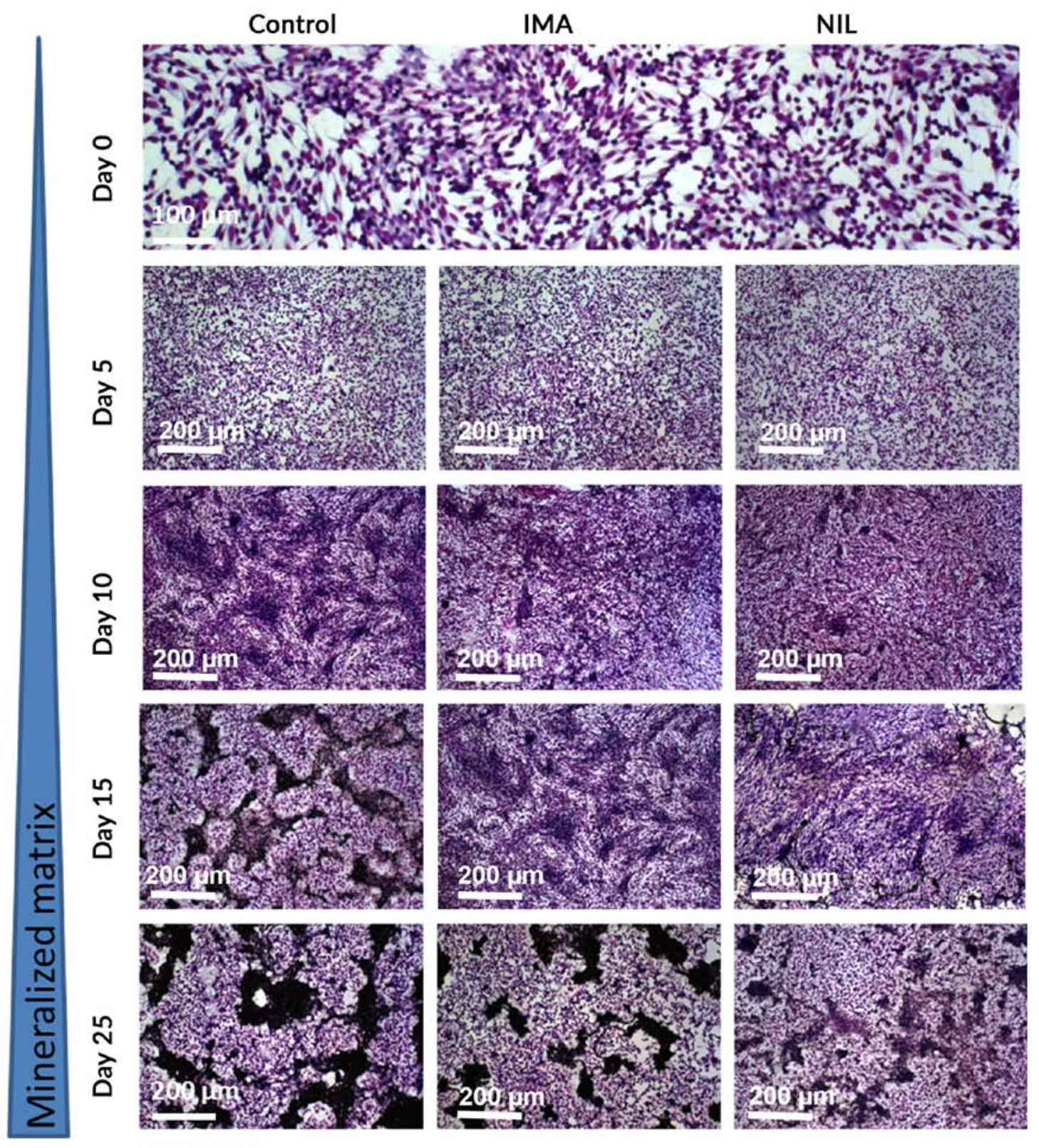

Mineralized matrix

Figure 1. Synthesis of mineralized bone matrix in the osteoblastic-like cell line SaOS-2 under treatment with IMA (middle column, $1 \mu \mathrm{M})$ and NIL (1 $\mu \mathrm{M}$, right-hand column) in comparison with untreated control (left-hand column): Hemofix-stained samples at different time points (day $0,5,10,15$ or 25$)$ of incubation. Mineralized areas are represented by black areas. Representative pictures are shown (n=3). IMA, imatinib; NIL, nilotinib.

5'-GCCAGTGGGAGATGTTAG-3', reverse: 5'-TTAGCT GCAAGTTTTCCC-3' (21); RANK, forward: 5'-AGGGAA AGCACTCACAGCTAAT-3', reverse: 5'-ACATGCTCCCTG CTGACC-3' (22); OPG, forward: 5'-GCTAACCTCACCTTC GAG-3', reverse: 5'-TGATTGGACCTGGTTACC-3' (21); ALP, forward: 5'-CAACCCTGGGGAGGAGAC-3', reverse: 5'-GCA TTGGTGTTGTACGTCTTG-3'; OSX, forward: 5'-CAAAGA AGCCGTACTCTGTGG-3', reverse: 5'-TGAAAGGAGCCC ATTAGTGC-3'; OCN, forward: 5'-TGAGAGCCCTCACAC TCCTC-3', reverse: 5'-ACCTTTGCTGGACTCTGCAC-3'; Wnt1, forward: 5'-CGCTGGAACTGTCCCACT-3', reverse: 5'-AACGCCGTTTCTCGACAG-3'; Wnt10b, forward: 5'-TTC TCTCGGGATTTCTTGGA-3', reverse: 5'-TCCGCTTCA
GGTTTTCAGTT-3'; GAPDH, forward: 5'-ACAGTCCAT GCCATCACTGCC-3', reverse: 5'-GCCTGCTTCACCACC TTCTTG-3' (23).

Statistical analysis. Statistical analyses at defined time points of incubation were performed using one-way analysis of variance and multiple comparisons were conducted using a post-hoc Bonferroni adjustment to evaluate the effects of IMA or NILtreated samples compared to untreated controls (GraphPad Prism v.6.0 software; GraphPad Software, Inc., La Jolla, CA, USA). Data are presented as means \pm standard deviation (SD). In all cases, $\mathrm{P}<0.05$ was considered to indicate a statistically significant difference. 


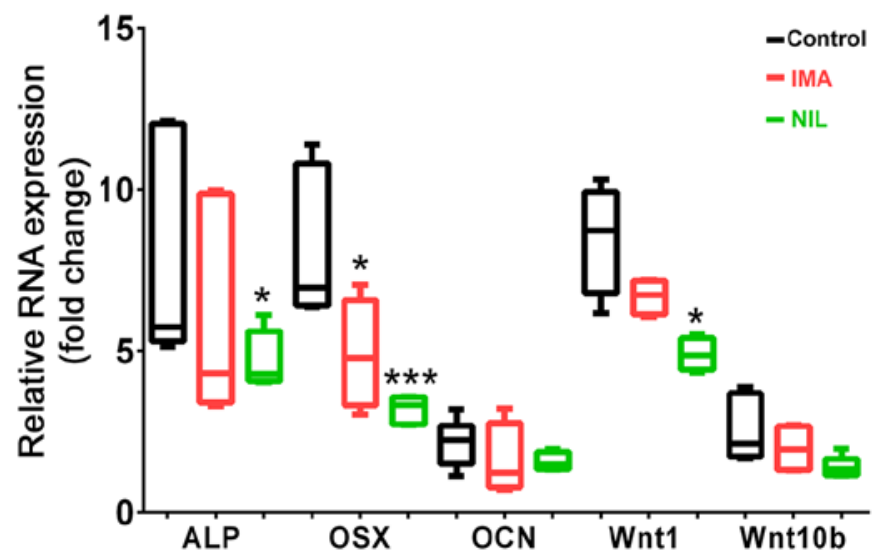

Figure 2. Gene expression levels of osteogenic markers in SaOS-2 cells treated for 25 days with IMA ( $1 \mu \mathrm{M}$, red bars) and NIL ( $1 \mu \mathrm{M}$, green bars) in comparison with untreated control (black bars). Reverse transcription-quantitative PCR results of ALP, OSX, OCN, Wnt1 and Wnt10b were normalized to GAPDH ( $n=4-6)$. ${ }^{*} \mathrm{P}<0.05$ and ${ }^{* * *} \mathrm{P}<0.001$, vs. control. IMA, imatinib; NIL, nilotinib; ALP, alkaline phosphatase; OSX, osterix; OCN, osteocalcin.

Mineralized matrix and immunofluorescence images quantified using ImageJ v.1.49 software (National Institute of Health, Bethesda, USA).

\section{Results}

TKIs inhibit osteoblast mineralization. SaOS-2 cells were cultured for up to 25 days in the presence of TKI [clinically effective concentration: $1 \mu \mathrm{M}(24,25)]$ and matrix mineralization was assessed at 5 day intervals. At day 25 , cultures treated with IMA exhibited $40 \%$ (a.u. $0.477 \pm 0.045$; Fig. 1) less mineralization than untreated controls (a.u. $0.788 \pm 0.053$; Fig. 1). This inhibitory effect was more pronounced with NIL, which reduced bone matrix by $90 \%$ (a.u. $0.081 \pm 0.01$; Fig. 1).

TKIs suppress the expression of osteogenic markers and Wnt molecules in $\mathrm{SaOS}-2$ cells. To study the mechanism underlying the reduced mineralization following TKI treatment, we investigated specific gene markers of osteoblastogenesis in SaOS-2 cells. After 25 days of IMA or NIL treatment, osterix (OSX) expression was significantly reduced by $3.2-$ and 4.9 -fold, respectively compared to the control (Fig. 2). IMA and NIL also reduced the gene expression of ALP by 2.3- and 3.4-fold, respectively, although only the latter was significant (Fig. 2). We also investigated the effects of TKIs on Wnt1 and Wnt10b expression, two members of the Wnt signaling pathway, which play a key role in maintaining bone homeostasis (26). NIL had a more pronounced effect on Wnt expression than IMA. In the presence of IMA, Wnt1 and Wnt10b mRNA levels were reduced by 1.8 - and 0.6 -fold, respectively, although these differences were not significant. By comparison, NIL reduced Wnt1 and Wnt10b expression by 3.6- $(\mathrm{P}<0.05)$ and 1.2 -fold, respectively.

TKIs increase RANKL/OPG ratio in osteoblasts. Finally, we investigated the expression levels of RANKL and OPG, which are involved in the coupling of bone formation and resorption. After 5 days of TKI treatment, we found no differences

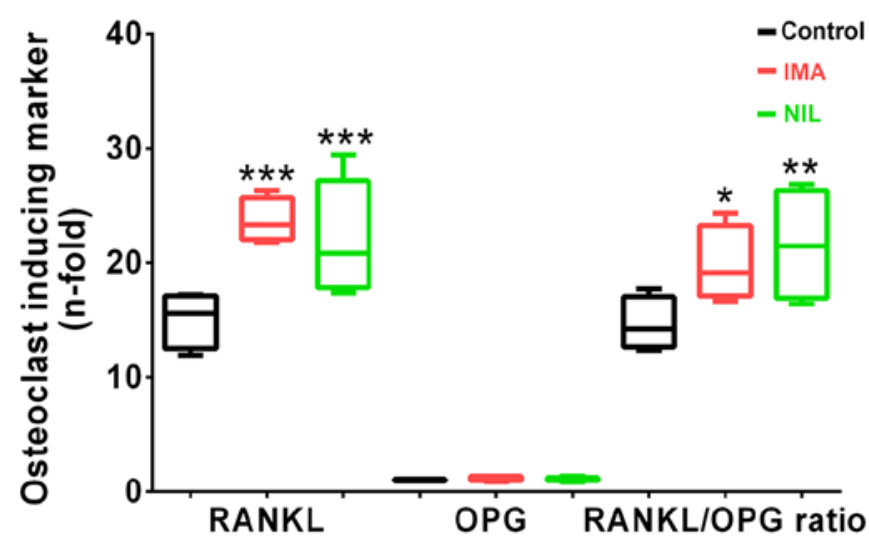

Figure 3. Gene expression of RANKL, OPG and RANKL/OPG ratio on day 25 of SaOS-2 cell treatment with IMA ( $1 \mu \mathrm{M}$, red bars) and NIL $(1 \mu \mathrm{M}$, green bars) in comparison with untreated control (black bars). Gene expression was normalized to GAPDH $(\mathrm{n}=4-6) .{ }^{*} \mathrm{P}<0.05,{ }^{* *} \mathrm{P}<0.01$ and ${ }^{* * *} \mathrm{P}<0.001$ vs.control. IMA, imatinib; NIL, nilotinib; RANKL, receptor activator of nuclear factor $\kappa \mathrm{B}$ ligand; $\mathrm{OPG}$, osteoprotegerin.

in RANKL or OPG gene expression levels. However, after 25 days, IMA and NIL significantly increased RANKL expression by 160 and $150 \%$, respectively, whereas OPG expression remained unchanged compared to controls. This consequently resulted in an increased RANKL/OPG ratio in osteoblasts treated with IMA $(+160 \%)$ and NIL $(+150 \%)$ (Fig. 3). These findings were verified by immunofluorescence staining of SaOS-2 cells reflected by medium fluorescence intensity (MFI) data, showing an increased fluorescence signal for RANKL under IMA (control: MFI/cell 0.71 $\pm 0,06$; IMA: MFI/cell 1.16 \pm 0.17 ) and NIL treatment (NIL: MFI/cell $2.48 \pm 0.15$ ) as well as a decreased fluorescence signal for OPG under IMA (control: MFI/cell 75.6 \pm 6.48 ; IMA: $\mathrm{MFI} /$ cell 35.4 \pm 4.82 ) and Nil (NIL: MFI/cell 37.4 \pm 7.16 ) treatment (Fig. 4).

\section{Discussion}

IMA and NIL were designed to inhibit BCR-ABL1, and emerged as powerful tools in the management of CML. However, the ATP binding domain in BCR-ABL1 that is targeted by IMA and NIL is structurally similar to other TKs, and consequently results in off-target effects. In CML patients receiving long-term IMA treatment, this has been reported to result in impaired skeletal growth. This is problematic because patients with CML need to remain on TKI therapy for longer durations to effectively eliminate all leukemic cell clones (27). Thus, for long-term safety, it is important to understand the effects of TKIs on bone remodeling.

In the current study, we report that TKI treatment decreased mineralization in osteoblastic cells, which was accompanied by decreased ALP and OSX expression. The effects on mineralization became apparent after 15 days of osteogenic induction in the presence of TKIs, but the maximal inhibitory effect was observed at day 25. Importantly, NIL inhibited osteoblastogenesis more potently than IMA. Our findings are consistent with a prior report which showed that 5 weeks of IMA treatment was associated with a significant reduction in bone 


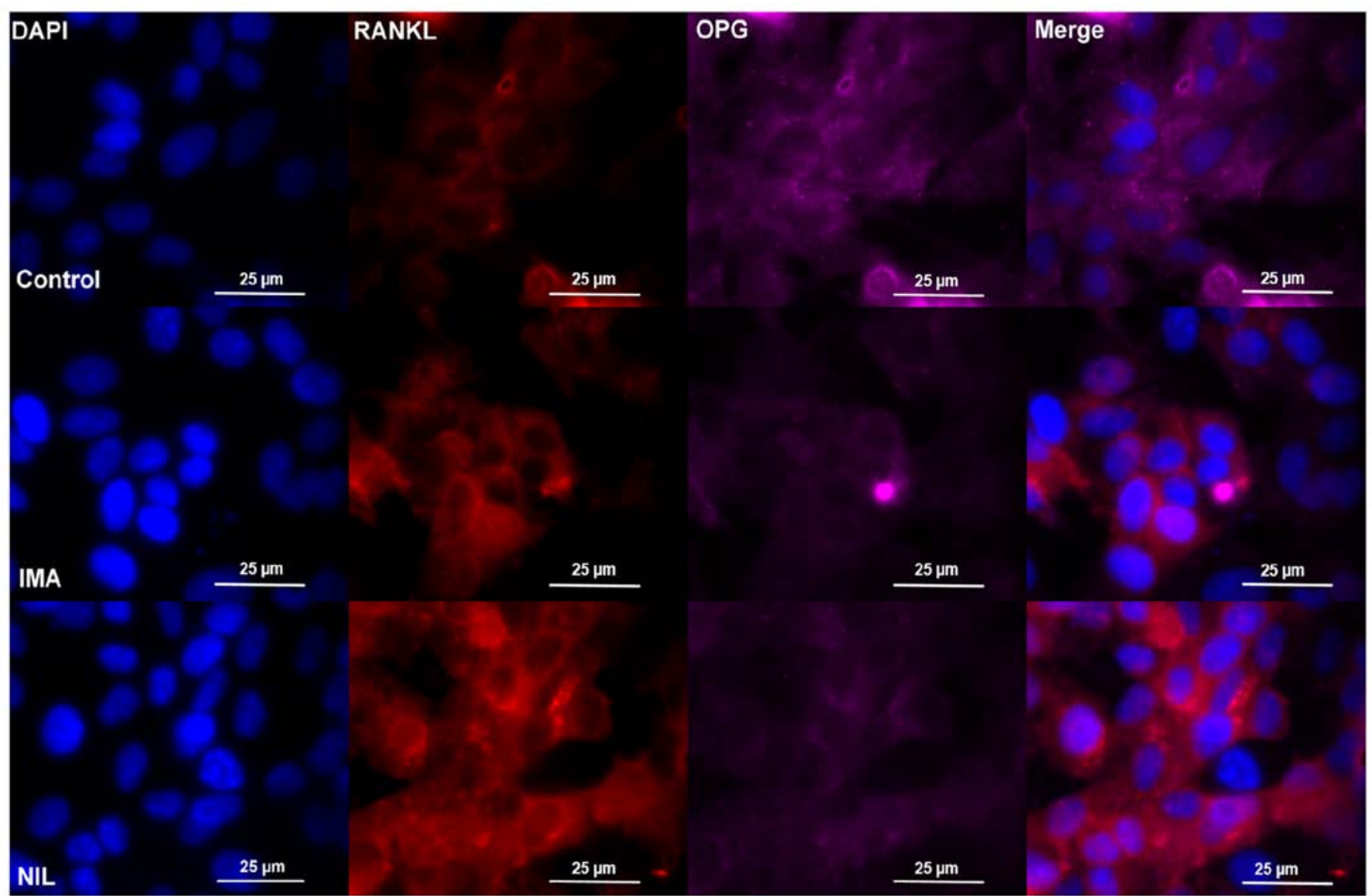

Figure 4. Immunofluorescence staining of SaOS-2 cells after incubation without TKI (control, upper row), IMA (1 $\mu \mathrm{M}$, middle row) or NIL ( $1 \mu \mathrm{M}$, lower row) for 25 days, respectively. Cells were stained with RANKL (red), OPG (purple), and DAPI (blue). One representative image is shown per treatment. Each experiment was performed three times. IMA, imatinib; NIL, nilotinib; RANKL, receptor activator of nuclear factor $\kappa \mathrm{B}$ ligand; OPG, osteoprotegerin.

mineral density in juvenile growing rats (24). We also found that both TKIs increased RANKL expression and decreased OPG levels, thereby resulting in an increased RANKL/OPG ratio. This increase in osteoclastogenic potential represents another mechanism by which TKIs may interfere with bone remodeling processes, which is consistent with prior reports by Tibullo et al $(28,29)$. In support of these findings at the clinical level, several groups have reported adverse effects of TKIs. A concentration of $1 \mu \mathrm{M}$ for both drugs were defined as clinically effective due to the level plasma concentration of IMA and NIL in human patients (25).

Within a few months of starting IMA treatment, adult CML patients displayed alternations in mineral metabolism (13), reduced bone formation and bone mass (30), and reduced OCN level compared to healthy controls (13).

However, the effects of TKIs on bone remain unresolved because there are several studies that have reported contradictory findings. In vitro, TKIs were reported to increase bone mineralization $(28,29)$, OPG levels $(31)$, and the expression of osteoblast specific genes $(15,28,32)$. In adult and pediatric patients, reduced as well as elevated osteocalcin levels has been described $(13,16,33,34)$ during TKI treatment. Considering these differences, it is pertinent to evaluate the design and context of these studies. There is substantial heterogeneity in choice of in vitro model system. Differences in cell lines (e.g., human vs. murine; primary cell vs. cell line; malignant vs. non-malignant) and assays may contribute to these discrepancies. Furthermore, the interpretation of clinical studies is complicated by inherent differences between adult and pediatric patients in which bone turnover varies substantially. In adult CML patients, TKI appears to promote bone formation, while in pediatric CML patients, TKI treatment decreases bone formation through growth retardation $(12,35,36)$.

We also found that the pro-osteogenic Wnt signaling pathway were down-regulated, specifically Wnt1. Wnt signaling is a key regulator of osteoblast function and bone homeostasis. Prior studies have shown that IMA reduces $\beta$-catenin expression, the main transcription factor for canonical Wnt signaling (37). Moreover, co-treatment of IMA with Wnt inhibitors potentiated the anti-leukemic effects of IMA (38). Thus, while suppression of Wnt signaling has beneficial effects on cancer progression, bone health may be compromised in the long-term.

Taken together, our study demonstrated that TKIs IMA and NIL negatively regulate osteoblast function in vitro. Moreover, TKI treatment was associated with an elevated RANKL/OPG ratio thereby, providing a pro-osteoclastogenic environment. Considering the previously described impact of TKIs on vitamin D metabolism $(17,18)$, which may further impair bone metabolism, patients on long-term TKI treatment should have their bone healthy regularly monitored. 


\section{Acknowledgements}

The authors of the present study would like to thank Dr. Ute Hempel (Institute of Physiological Chemistry, Medical Faculty Carl Gustav Carus, Technische Universität Dresden, Dresden, Germany) for supplying SaOS-2 cells as well as the helpful suggestions and handling advice. The authors would also like to thank Novartis International AG (Basel, Switzerland) for supplying TKIs and for their financial support.

\section{Funding}

The present study was supported by Novartis International AG (grant no. HTAS-079).

\section{Availability of data and materials}

The datasets used and/or analyzed during the present study are available from the corresponding author on reasonable request.

\section{Authors' contributions}

AB, LMK, MS, SB and MR conceived and designed the study. LMK managed the data and the overall study, verified the results and prepared the visual aspects of the work. JTT and LMK analyzed the data. AB and LMK acquired the funding. LMK, AW and SIK performed the experiments. JTT, MS, SIK and MR provided the reagents, materials, computing resources and analytical tools. AB, MS, MR and SB supervised the study. LMK and JTT wrote the manuscript. JTT, LMK, MS and MR critically analyzed the manuscript for important intellectual content. All authors have read and approved the final version.

\section{Ethics approval and consent to participate}

Not applicable.

\section{Patient consent for publication}

Not applicable.

\section{Competing interests}

The two TKIs used in the present study, IMA and NIL, were obtained from Novartis International AG (Basel, Switzerland) who also financially supported the study.

\section{References}

1. Champagne MA, Capdeville R, Krailo M, Qu W, Peng B, Rosamilia M, Therrien M, Zoellner U, Blaney SM and Bernstein M; Children's Oncology Group phase 1 study: Imatinib mesylate (STI571) for treatment of children with Philadelphia chromosome-positive leukemia: Results from a children's oncology group phase 1 study. Blood 104: 2655-2660, 2004.

2. Druker BJ, Tamura S, Buchdunger E, Ohno S, Segal GM, Fanning S, Zimmermann J and Lydon NB: Effects of a selective inhibitor of the Abl tyrosine kinase on the growth of Bcr-Abl positive cells. Nat Med 2: 561-566, 1996.

3. Druker BJ, Talpaz M, Resta DJ, Peng B, Buchdunger E, Ford JM, Lydon NB, Kantarjian H, Capdeville R, Ohno-Jones S and Sawyers CL: Efficacy and safety of a specific inhibitor of the BCR-ABL tyrosine kinase in chronic myeloid leukemia. N Engl J Med 344: 1031-1037, 2001.
4. Grigg A and Hughes T: Role of allogeneic stem cell transplantation for adult chronic myeloid leukemia in the imatinib era. Biol Blood Marrow Transplant 12: 795-807, 2006.

5. Millot F, Guilhot J, Nelken B,Leblanc T, De BontES, Bekassy AN, Gadner H, Sufliarska S, Stary J, Gschaidmeier H, et al: Imatinib mesylate is effective in children with chronic myelogenous leukemia in late chronic and advanced phase and in relapse after stem cell transplantation. Leukemia 20: 187-192, 2006.

6. Roy L, Guilhot J, Krahnke T, Guerci-Bresler A, Druker BJ, Larson RA, O'Brien S, So C, Massimini G and Guilhot F: Survival advantage from imatinib compared with the combination interferon-alpha plus cytarabine in chronic-phase chronic myelogenous leukemia: Historical comparison between two phase 3 trials. Blood 108: 1478-1484, 2006.

7. Iijima R, Byrne RA,Dibra A,Ndrepepa G, Spaulding C,Laarman GJ, Menichelli M, Valgimigli M, Di Lorenzo E, Kaiser C, et al: Drug-eluting stents versus bare-metal stents in diabetic patients with ST-segment elevation acute myocardial infarction: A pooled analysis of individual patient data from seven randomized trials. Rev Esp Cardiol 62: 354-364, 2009 (In English, Spanish).

8. Hobernicht SL, Schweiger B, Zeitler P, Wang M and Hunger SP: Acquired growth hormone deficiency in a girl with chronic myelogenous leukemia treated with tyrosine kinase inhibitor therapy. Pediatr Blood Cancer 56: 671-673, 2011

9. Deguchi Y, Kimura S, Ashihara E, Niwa T, Hodohara K, Fujiyama Y and Maekawa T: Comparison of imatinib, dasatinib, nilotinib and INNO-406 in imatinib-resistant cell lines. Leuk Res 32: 980-983, 2008.

10. Kantarjian H, Giles F, Wunderle L, Bhalla K, O'Brien S, Wassmann B, Tanaka C, Manley P, Rae P, Mietlowski W, et al: Nilotinib in imatinib-resistant CML and Philadelphia chromosome-positive ALL. N Engl J Med 354: 2542-2551, 2006.

11. Jabbour E, El AS, Cortes J and Kantarjian H: Nilotinib: A novel Bcr-Abl tyrosine kinase inhibitor for the treatment of leukemias. Expert Opin Investig Drugs 17: 1127-1136, 2008.

12. Shima H, Tokuyama M, Tanizawa A, Tono C, Hamamoto $\mathrm{K}$, Muramatsu H, Watanabe A, Hotta N, Ito M, Kurosawa H, et al: Distinct impact of imatinib on growth at prepubertal and pubertal ages of children with chronic myeloid leukemia. J Pediatr 159: 676-681, 2011.

13. Berman E, Nicolaides M, Maki RG, Fleisher M, Chanel S, Scheu K, Wilson BA, Heller G and Sauter NP: Altered bone and mineral metabolism in patients receiving imatinib mesylate. N Engl J Med 354: 2006-2113, 2006.

14. Fierro F, Illmer T, Jing D, Schleyer E, Ehninger G, Boxberger S and Bornhäuser $\mathrm{M}$ : Inhibition of platelet-derived growth factor receptorbeta by imatinib mesylate suppresses proliferation and alters differentiation of human mesenchymal stem cells in vitro. Cell Prolif 40: 355-366, 2007

15. Fitter S, Dewar AL, Kostakis P, To LB, Hughes TP, Roberts MM, Lynch K, Vernon-Roberts B and Zannettino AC: Long-term imatinib therapy promotes bone formation in CML patients. Blood 111: 2538-2547, 2008.

16. Jaeger BA, Tauer JT, Ulmer A, Kuhlisch E, Roth HJ and Suttorp M: Changes in bone metabolic parameters in children with chronic myeloid leukemia on imatinib treatment. Med Sci Monit 18: CR721-CR728, 2012.

17. Kroschwald L, Suttorp M, Tauer JT, Zimmermann N, Gunther C and Bauer A: Offtarget effect of imatinib and nilotinib on human vitamin D3 metabolism. Mol Med Rep 17: 1382-1388, 2018.

18. Mehlig LM, Garve C, Tauer JT, Suttorp M and Bauer A: Inhibitory effects of imatinib on vitamin $\mathrm{D}(3)$ synthesis in human keratinocytes. Mol Med Rep 11: 3143-3147, 2015.

19. Boyce BF and Xing L: Biology of RANK, RANKL, and osteoprotegerin. Arthritis Res Ther 9 (Suppl 1): S1, 2007.

20. Schindelin J, Arganda-Carreras I, Frise E, Kaynig V, Longair M, Pietzsch T, Preibisch S, Rueden C, Saalfeld S, Schmid B, et al: Fiji: An open-source platform for biological-image analysis. Nat Methods 9: 676-682, 2012.

21. Mori K, Le GB, Berreur M, Riet A, Moreau A, Blanchard F, Chevalier C, Guisle-Marsollier I, Léger J, Guicheux J, et al: Human osteosarcoma cells express functional receptor activator of nuclear factor-kappa B. J Pathol 211: 555-562, 2007.

22. Xiong MY, Liu LQ, Liu SQ, Liu ZH and Gao HF: Effects of osteoprotegerin, RANK and RANKL on bone destruction and collapse in avascular necrosis femoral head. Am J Transl Res 8: 3133-3140, 2016.

23. Stephens AS, Stephens SR and Morrison NA: Internal control genes for quantitative RT-PCR expression analysis in mouse osteoblasts, osteoclasts and macrophages. BMC Res Notes 4: 410, 2011. 
24. Tauer JT, Hofbauer LC, Jung R, Gerdes S, Glauche I, Erben RG and Suttorp M: Impact of long-term exposure to the tyrosine kinase inhibitor imatinib on the skeleton of growing rats. PLoS One 10: e0131192, 2015.

25. Gandia P,Arellano C,Lafont T,Huguet F, Malard L and Chatelut E: Should therapeutic drug monitoring of the unbound fraction of imatinib and its main active metabolite N-desmethyl-imatinib be developed? Cancer Chemother Pharmacol 71: 531-536, 2013.

26. Baron R and Kneissel M: WNT signaling in bone homeostasis and disease: From human mutations to treatments. Nat Med 19 179-192, 2013.

27. Suttorp M, Schulze P, Glauche I, Gohring G, von Neuhoff $N$ Metzler M, Sedlacek P, de Bont ESJM, Balduzzi A, Lausen B, et al: Front-line imatinib treatment in children and adolescents with chronic myeloid leukemia: Results from a phase III trial. Leukemia 32: 1657-1669, 2018.

28. Tibullo D, Giallongo C, La CP, Berretta S, Stagno F, Chiarenza A Conticello C,Palumbo GA and Di Raimondo F: Effects of imatinib mesylate in osteoblastogenesis. Exp Hematol 37: 461-468, 2009.

29. Tibullo D, Barbagallo I, Giallongo C, La CP, Branca A, Conticello C, Stagno F, Chiarenza A, Palumbo GA and Di Raimondo F: Effects of second-generation tyrosine kinase inhibitors towards osteogenic differentiation of human mesenchymal cells of healthy donors. Hematol Oncol 30: 27-33, 2012.

30. O'Sullivan S, Naot D, Callon KE, Watson M, Gamble GD Ladefoged M, Karsdal MA, Browett P, Cornish J and Grey A: Imatinib mesylate does not increase bone volume in vivo. Calcif Tissue Int 88: 16-22, 2011.

31. O'Sullivan S, Tay ML, Lin JM, Bava U, Callon K, Cornish J, Naot D and Grey A: Tyrosine kinase inhibitors regulate OPG through inhibition of PDGFR $\beta$. PLoS One 11: e0164727, 2016.
32. O'Sullivan S, Naot D, Callon K, Porteous F, Horne A, Wattie D, Watson M, Cornish J, Browett P and Grey A: Imatinib promotes osteoblast differentiation by inhibiting PDGFR signaling and inhibits osteoclastogenesis by both direct and stromal cell-dependent mechanisms. J Bone Miner Res 22: 1679-1689, 2007.

33. O'Sullivan S, Horne A, Wattie D, Porteous F, Callon K, Gamble G, Ebeling P, Browett P and Grey A: Decreased bone turnover despite persistent secondary hyperparathyroidism during prolonged treatment with imatinib. J Clin Endocrinol Metab 94: 1131-1136, 2009.

34. Vandyke K, Fitter S, Dewar AL, Hughes TP and Zannettino AC: Dysregulation of bone remodeling by imatinib mesylate. Blood 115: 766-774, 2010.

35. Narayanan KR, Bansal D, Walia R, Sachdeva N, Bhansali A, Varma N and Marwaha RK: Growth failure in children with chronic myeloid leukemia receiving imatinib is due to disruption of GH/IGF-1 axis. Pediatr Blood Cancer 60: 1148-1153, 2013

36. Millot F, Guilhot J, Baruchel A, Petit A, Leblanc T, Bertrand Y, Mazingue F, Lutz P, Vérité C, Berthou C, et al: Growth deceleration in children treated with imatinib for chronic myeloid leukaemia. Eur J Cancer 50: 3206-3211, 2014.

37. Zhou L, An N, Haydon RC, Zhou Q, Cheng H, Peng Y, Jiang W, Luu HH, Vanichakarn P, Szatkowski JP, et al: Tyrosine kinase inhibitor STI-571/Gleevec down-regulates the beta-catenin signaling activity. Cancer Lett 193: 161-170, 2003.

38. Suknuntha K, Thita T, Togarrati PP, Ratanachamnong P, Wongtrakoongate P, Srihirun S, Slukvin I and Hongeng S: Wnt signaling inhibitor FH535 selectively inhibits cell proliferation and potentiates imatinib-induced apoptosis in myeloid leukemia cell lines. Int J Hematol 105: 196-205, 2017. 